\title{
Plantes médicinales de l'Afrique de l'Ouest pour la prise en charge des affections respiratoires pouvant se manifester au cours de la Covid-19
}

\author{
Mahamane HAIDARA ${ }^{1 *}$, Mamadou Lamine DIARRA ${ }^{1}$, Sékou DOUMBIA ${ }^{1}$, Adama \\ DENOU $^{1}$, Daouda DEMBELE ${ }^{1}$, Birama DIARRA ${ }^{2}$ et Rokia SANOGO ${ }^{1,2}$ \\ ${ }^{l}$ Faculté de Pharmacie, Université des Sciences des Techniques et des Technologies de Bamako (USTTB), \\ BP 1805 Mali. \\ ${ }^{2}$ Département Médecine Traditionnelle, Bamako, BP 1746 Mali. \\ *Auteur correspondant ; E-mail : mahamanehaidara83@gmail.com
}

\section{RESUME}

Dans le cadre de contribuer à la prise en charge symptomatique des affections respiratoires pouvant se manifester pendant la Covid-19, la présente étude a été entreprise pour recenser les plantes médicinales Africaines actives contre ces manifestations. Une étude bibliographique a été effectuée pour recenser les espèces végétales utilisées dans la prise en charge des maladies respiratoires en Afrique de l'Ouest. La fréquence de citation et un score de convergence d'usage des espèces ont été également calculés. Un total de 318 recettes issues de 145 espèces végétales a été inventorié à travers différentes publications. Guiera senegalensis, Sterculia setigera, Bridelia ferruginea, Euphorbia hirta et Terminalia avicennioides étaient les plantes les plus citées avec les meilleurs scores de convergence d'usage. Les feuilles de ces espèces étaient les plus citées tandis que la toux était la plus indiquée. Les résultats de cette étude pourraient servir de base de données des espèces végétales utilisées dans la prise en charge des maladies du tractus respiratoire en Afrique de l'ouest.

(C) 2020 International Formulae Group. All rights reserved.

Mots clés : Plantes médicinales, Affections respiratoires, Covid-19, Afrique de l'Ouest.

\section{Medicinal plants from West Africa for the management of respiratory diseases that may appear during the Covid-19}

\begin{abstract}
As part of contributing to the symptomatic management of respiratory conditions that may manifest during Covid-19, the current study was undertaken to identify African medicinal plants active against these manifestations. A bibliographic study was carried out to identify the plant species used in the management of respiratory diseases in West Africa. The frequency of citation and a convergence score for the use of the species were also calculated. A total of 318 recipes from 145 plant species were inventoried through various publications. Guiera senegalensis, Sterculia setigera, Bridelia ferruginea, Euphorbia hirta and Terminalia avicennioides were the most cited plants with the best usage convergence scores. Leaves from these species were most cited while the cough was more indicated. The results of this study could serve as a database of plant species used in the management of respiratory tract diseases in West Africa.
\end{abstract}

(C) 2020 International Formulae Group. All rights reserved.

Keywords: Medicinal plants, Respiratory Affections, Covid-19, West Africa. 


\section{INTRODUCTION}

A travers l'histoire, les plantes issues des pharmacopées traditionnelles ont été sources de traitements et de principes actifs qui ont contribué à la lutte contre les maladies. A titre d'exemple, les deux principes actifs (quinine et artémisinine), utilisés dans la prise en charge du paludisme sont issus de pharmacopées traditionnelles de l'Amérique du Sud et de la chine.

La stratégie de la région africaine de l'OMS/AFRO (2000) a encouragé l'intégration dans les systèmes de santé des pratiques médicales et des remèdes traditionnels qui ont donné la preuve de leur innocuité, de leur efficacité et de leur qualité. En Afrique de l'Ouest, l'Organisation Ouest Africaine de Santé (OOAS) a publié en 2013 la Pharmacopée de l'Afrique de l'Ouest (PAO1) qui comprend les monographies de 54 plantes médicinales les plus répandues dans les pays membres de la Communauté Economique des Etats de l'Afrique de l'Ouest (CEDEAO), utilisées pour le traitement du paludisme, VIH SIDA, tuberculose, diabète, hypertension artérielle (HTA) et la drépanocytose (Organisation Ouest Africaine de la Santé, 2013).

Ces monographies constituent des sources d'informations précieuses ethnobotaniques, de données sûres, efficace, de qualité et de molécules actives pour la prise en charge de pathologies majeures (Organisation Ouest Africaine de la Santé, 2013). Une exploitation judicieuse de ces données peut contribuer à la mise au point de médicaments utilisables dans le traitement des pathologies fréquentes et émergentes.

En Chine, Artemisia annua a joué un rôle particulier dans la lutte contre le coronavirus car elle est administrée seule et non pas mélangée à d'autres plantes. Elle est utilisée pour résoudre les symptômes de difficulté respiratoire modérée. C'est une plante qui est sure et efficace (World Health Organization, 2004; Van der Kooyn et Sullivan, 2013). Conformément, à l'expérience de la Chine, en Afrique des médicaments à base de plantes avec autorisation de mise sur le marché, peuvent contribuer à la prise en charge des difficultés respiratoires et de la toux. Pour la toux, le sirop Balembo ${ }^{\circledR}$ est produit à base de Crossopteryx febrifuga Benth au Mali, le sirop Elooko $^{\circledR}$ à base de Guiera senegalensis produit au Sénégal, le sirop Douba ${ }^{\circledR}$ à base de Entada africana est produit au Burkina Faso et le sirop Dissotis ${ }^{\circledR}$ à base de Dissotis rotundifolia est produit en Guinée (Pousset, 2006). Au Mali, le sirop Balembo ${ }^{\circledR}$ a été proposé dans la prise en charge de la toux sèche chez les personnes atteintes de Covid-19. Cela a été possible dans ta le cadre d'un partenariat avec le Conseil National de l'Ordre des Pharmaciens (CNOP) du Mali.

Dans le but de contribuer à la lutte contre les affections respiratoires pouvant se manifester lors d'une infection à Covid-19, la présente étude a été initiée avec comme objectif d'inventorier les plantes médicinales utilisées dans la prise en charge des maladies respiratoires en Afrique de l'Ouest (Benin, Burkina-Faso, Cap-Vert, Côte d'Ivoire, Gambie, Ghana, Guinée, Guinée-Bissau, Liberia, Mali, Mauritanie, Niger, Nigeria, Sénégal, Sierra Leone et Togo). Les résultats de cette étude, pourront permettre d'une part à identifier d'autres plantes «candidates» pour la mise au point de nouveau médicaments traditionnels améliorés (MTA) dans la prise en charge des maladies respiratoires et d'autre part de constituer une base de données des plantes utilisées dans le traitement de ces maladies en Afrique de l'Ouest.

\section{COLLECTE DES DONNEES}

Une étude bibliographique exhaustive a été effectuée pour recenser les plantes utilisées dans la prise en charge des maladies respiratoires en consultant la base de données Google Scholar. Les mots clés tels que «plantes utilisées dans le traitement des maladies respiratoires en Afrique de l'Ouest» ou «medicinal plants used for the treatment of respiratory diseases in West Africa » ont été utilisés. La présente investigation a porté sur des articles originaux d'études 
ethnobotaniques, pharmacologiques et toxicologiques couplés à une revue de littérature.

Les informations relevées portaient surtout sur le nom des plantes utilisées dans la prise en charge des maladies respiratoires, les parties utilisées, le mode de préparation et la posologie si ceux-ci étaient mentionnés.

\section{ANALYSES DES DONNEES}

Les données ont été saisies et analysées avec le logiciel Microsoft Excel version 10. La fréquence de citation $(\mathrm{Fc})$ de chaque plante a été calculée selon la formule décrite par Guinnin et al. (2015).

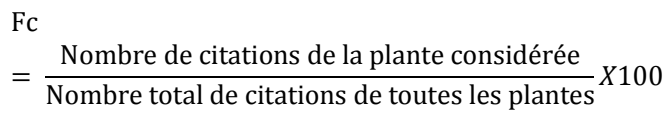

Un score (S) de convergence d'usage bibliographique et de diffusion géographique (Chassagne, 2017) a été aussi calculé selon la formule suivante :

$$
\mathrm{S}=\mathbf{I 1} \times \mathbf{I} 2
$$

I1 : Nombre de publications (extraits de la revue bibliographique) qui mentionne l'usage de l'espèce dans la prise en charge des maladies respiratoires, et $\mathbf{2}$ : Nombre des pays dans lequel est reporté l'usage de cette espèce pour la pathologie donnée.

\section{LES ESPECES VEGETALES INVENTORIEES}

$\mathrm{Au}$ total 12 références ont été consultées dont neuf articles d'études ethnobotaniques (Koné et al., 2002 ; Mann et al., 2007; Zerbo et al., 2007 ; Ajibesin et al., 2008 ; Nadembega et al., 2011 ; Adomou et al., 2012 ; Yapi et al., 2015 ; Béné et al., 2016 ; Diop et al., 2016), un article d'étude pharmacologique (Yeo et al., 2008), un article d'étude toxicologique (Irie-N'guessan et al., 2011) et un article d'étude bibliographique (Adesina et al., 2017). Six pays reportent l'utilisation des espèces végétales dans la prise en charge des maladies respiratoires en Afrique de l'Ouest. Ces pays sont : le Benin, le Burkina
Faso, la Cote d'Ivoire, le Mali, le Nigeria et le Sénégal.

Un total de trois cent dix-huit (318) recettes issues de cent quarante-cinq (145) espèces végétales ont été inventoriées. Les espèces végétales les plus citées étaient Guiera senegalensis (4 citations), Sterculia setigera, Bridelia ferruginea, Euphorbia hirta et Terminalia avicennioides avec 3 citations chacune. Les meilleurs scores d'usages ont été obtenus aussi avec ces espèces: Guiera senegalensis ( 4 références provenant de 4 pays, $\mathrm{S}=16)$, Sterculia setigera (3 références provenant de 3 pays, $S=9$ ), Bridelia ferruginea, Euphorbia hirta et Terminalia avicennioides (3 références provenant de 2 pays, $S=6$ ).

Ces résultats suggèrent une forte convergence d'utilisation de ces espèces végétales dans la prise en charge des maladies respiratoires (Chassagne, 2017).

\section{LES PARTIES DES PLANTES UTILISEES DANS LA PREPARATION DES RECETTES}

Les feuilles $(24,2 \%)$ suivies des écorces de tronc $(17,6 \%)$ et des racines $(16,7 \%)$ sont les organes de plante les plus employés dans la préparation des recettes (Tableau 1). Par ailleurs l'équipe de Bahmani avait aussi révélé que les feuilles étaient majoritairement utilisées avec $24 \%$ chez les tradipraticiens pour traiter les maladies infectieuses en Iran (Bahmani et al., 2015). La forte fréquence d'utilisation de feuilles peut être expliquée par l'aisance et la rapidité de la récolte mais aussi par le fait qu'elles sont le siège de la photosynthèse et donc souvent lieu de stockage des métabolites secondaires responsables des propriétés biologiques de la plante (Jdaidi et Hasnaoui, 2016). En plus Hossain et ses collèges ont rapporté que de nombreux métabolites secondaires sont doués d'effets antioxydants qui sont essentiels pour bloquer la pathogenèse de plusieurs maladies et le processus du vieillissement causé par les radicaux libres (Hossain et al., 2017). 
Tableau 1 : Fréquence des parties des espèces végétales utilisées dans la préparation des recettes.

\begin{tabular}{lcc}
\hline Partie utilisée & Nombre de citation & Pourcentage \\
\hline Feuille & 77 & 24,2 \\
Ecorce de tronc & 56 & 17,6 \\
Racine & 53 & 16,7 \\
Non précisé & 45 & 14,2 \\
Plante entière & 19 & 6,0 \\
Fruit & 15 & 4,7 \\
Fleur & 7 & 2,2 \\
Partie aérienne & 7 & 2,2 \\
Branche & 6 & 1,9 \\
Noix & 6 & 1,9 \\
Graine & 5 & 1,6 \\
Gui & 5 & 1,6 \\
Rhizome & 5 & 1,6 \\
Fruit + Feuille & 4 & 1,3 \\
Galle & 2 & 0,6 \\
Pulpe & 2 & 0,6 \\
Bulbe & 1 & 0,3 \\
Feuille + Graines & 1 & 0,3 \\
Germe de blé & 1 & 0,3 \\
Gomme & 1 & 0,3 \\
Total & $\mathbf{3 1 8}$ & $\mathbf{1 0 0}$ \\
\hline
\end{tabular}

\section{LES INDICATIONS SPECIFIQUES DES ESPECES VEGETALES DANS LES MALADIES RESPIRATOIRES}

Les indications spécifiques des espèces végétales inventoriées sont la toux, la tuberculose, l'asthme, la bronchite, l'irritation de la gorge, la pneumonie, le rhume, et la coqueluche. La toux était l'indication la plus citée des recettes $(48,7 \%)$ et des espèces de plantes $(70,3 \%)$ (Tableau 2).

Guiera senegalensis : Les feuilles, les racines et la galle sont utilisées dans le traitement de la toux (Nadembega et al., 2011 ; Diop et al., 2016) et de la tuberculose (Nadembega et al., 2011). Selon Adesina et al. (2017), Guiera senegalensis est aussi utilisé dans le traitement de la bronchite et du rhume.
De nombreuses études pharmacologiques ont été menées sur Guiera senegalensis afin de valider ces nombreux usages traditionnels.

Les alcaloïdes totaux $(15,25$, et 30 $\mathrm{mg} / \mathrm{kg}$ per os) de Guiera senegalensis ont démontré aussi une activité antitussive comparable à celle de la codéine $(50 \mathrm{mg} / \mathrm{kg})$ sur un modèle de toux induit par l'ammoniaque chez des cobayes (Diatta et al., 2007). L'activité bronchodilatatrice des feuilles a été évaluée sur un modèle de bronchoconstriction induite par l'histamine et l'acétylcholine ex vivo sur des organes isolés des cobayes. L'extrait éthanolique $(4-8 \mathrm{mg} / \mathrm{ml})$ a diminué la bronchoconstriction (Chedi et Mandawari, 2018). 
Tableau 2 : Fréquence des recettes et des plantes utilisées dans les maladies respiratoires.

\begin{tabular}{lcccc}
\hline Indications & $\begin{array}{c}\text { Nombre de } \\
\text { recette }\end{array}$ & $\begin{array}{c}\text { Pourcentage de } \\
\text { recette }\end{array}$ & $\begin{array}{c}\text { Nombre de } \\
\text { plante }\end{array}$ & Pourcentage de plante \\
\hline Toux & 155 & 48,7 & 102 & 70,3 \\
Tuberculose & 59 & 18,6 & 41 & 28,3 \\
Asthme & 42 & 13,2 & 38 & 26,2 \\
Bronchite & 23 & 7,2 & 17 & 11,7 \\
Irritation de la gorge & 15 & 4,7 & 10 & 6,9 \\
Pneumonie & 12 & 3,8 & 10 & 6,9 \\
Rhume & 9 & 2,8 & 8 & 5,5 \\
Coqueluche & 3 & 0,9 & 3 & 2,1 \\
Total & $\mathbf{3 1 8}$ & $\mathbf{1 0 0}$ & & \\
\hline
\end{tabular}

Les propriétés antivirales (Lamien et al., 2005 ; Arbab et al., 2017 ; Parvez et al., 2018 ;), antibactériennes (Tine et al., 2019), antalgiques (Jigam et al., 2011), antipyrétiques (Ahmed et al., 2018) et antiinflammatoires (Nwafor et Hamza, 2007) ont été aussi rapportées dans la littérature.

Ces propriétés antitussives, bronchodilatatrices, antiinfectieuses, antalgiques, antipyrétiques et antiinflammatoires de Guiera senegalensis démontrées pourraient justifier son utilisation dans le traitement de la toux, de la bronchite, de la tuberculose et du rhume.

Sterculia setigera : La décoction des écorces de tronc est utilisée dans le traitement de la toux (Mann et al., 2007 ; Zerbo et al., 2007) et de la bronchite (Mann et al., 2007 ; Diop et al., 2016). Celle de la racine est utilisée contre la bronchite (Diop et al., 2016). Selon les données de la littérature, l'extrait méthanolique des écorces de tronc de Sterculia setigera est doué de propriétés antimicrobienne (Tor-Anyiin et al., 2011). Babalola et al. (2012) ont démontré les propriétés antiMycobacterium tuberculosis des extraits des feuilles. Ces propriétés antimicrobiennes pourraient justifier son utilisation dans le traitement de la toux et de la bronchite.
Bridelia ferruginea : L'infusion de la racine est utilisée dans le traitement de la toux (Koné et al., 2002). L'écorce de tronc est utilisée contre la coqueluche (Mann et al., 2007). Selon Adesina et al. (2017), Bridelia ferrugina est utilisé aussi dans la prise en charge de l'asthme. Des travaux antérieurs ont permis de démontrer les propriétés antimicrobiennes (Adebayo et Ishola, 2009; Jose et Kayode, 2009 ; Owoseni et al., 2010 ; Alowanou et al., 2015 ; Luka et al., 2020) et antiinflammatoires (Alowanou et al., 2015) des extraits de Bridelia ferruginea. Ces propriétés pourraient justifier son utilisation dans le traitement de la toux et de l'asthme.

Euphorbia hirta : La décoction de la plante entière est utilisée dans la prise en charge de la toux (Mann et al., 2007), de l'asthme (Mann et al., 2007 ; Ajibesin et al., 2008) et du rhume (Diop et al., 2016). Des études antérieures ont démontré les propriétés antibactériennes (Gyuris et al., 2009 ; Kumar et al., 2010; Ndhlala et al., 2013; Al-Snafi, 2017), antivirales (Ndhlala et al., 2013 ; AlSnafi, 2017 ; Al-Snafi, 2019), antalgiques, antiinflammatoires et antipyrétiques (Kumar et al., 2010 ; Al-Snafi, 2017). Les propriétés antiasthmatiques des extraits et d'un triterpenoïde isolé des tiges ont été aussi 
rapportées (Kumar et al., 2010; Prachi et Pradeep, 2014 ; Al-Snafi, 2017). Ces propriétés pourraient justifier son utilisation dans le traitement de la toux et de l'asthme.

Terminalia avicennioides: Les feuilles sont utilisées contre la toux (Zerbo et al., 2007) et la bronchite (Mann et al., 2007), les écorces de tronc et les guis sont utilisés contre la bronchite et la tuberculose (Mann et al., 2007). Des études antérieures ont démontré les propriétés antimicrobiennes des extraits de Terminalia avicennioides in vitro sur des bactéries isolées des patients souffrant de complication des maladies respiratoires (Mann et al., 2008a ; Mann, 2012). L'activité antiMycobacterium tuberculosis des extraits ou des triterpenoïdes isolés des écorces de racine a été démontrée (Mann et al., 2008b ; Mann et al., 2009 ; Mann et al., 2012). Les flavonoïdes et les saponosides isolées des écorces de racine ont montré aussi une activité antimicrobienne (Usman et al., 2018). Ces propriétés antimicrobiennes pourraient justifier son utilisation dans le traitement de la toux, de la bronchite, et de la tuberculose.

\section{Conclusion}

Il ressort de cette étude qu'en Afrique de l'Ouest, de nombreuses espèces de plante sont utilisées dans la prise en charge des maladies respiratoires, ce qui pourra offrir l'opportunité d'une éventuelle utilisation contre les manifestations respiratoires liées à la Covid-19. Guiera senegalensis, Sterculia setigera, Euphorbia hirta, Bridelia ferruginea et Terminalia avicennioides peuvent être des «candidates» potentielles pour la mise au point de nouveaux MTA utilisés dans la prise en charge des maladies respiratoires.

Les résultats de cette étude pourraient aussi servir à constituer une base de données des espèces de plante utilisées dans la prise en charge des maladies respiratoires en Afrique de l'Ouest.

\section{CONFLIT D'INTERETS}

Les auteurs déclarent une absence de conflit d'intérêts.

\section{CONTRIBUTIONS DES AUTEURS}

MH a participé à la collecte des documents et l'analyse des données et à la rédaction du manuscrit. MLD a participé à l'analyse des données et à la rédaction de la publication. $\mathrm{SD}, \mathrm{AD}, \mathrm{DD}$ et $\mathrm{BD}$ ont participé à la correction du manuscrit. RS a conçu l'étude et a participé à la correction du manuscrit.

\section{REFERENCES}

Adebayo EA, Ishola OR. 2009. Phytochemical and antimicrobial screening of the crude extracts from the root, stem bark and leaves of Bridelia ferruginea. African Journal of Biotechnology, 8(4): 650-653.

Adesina SK, Johnny II, Olayiwola G. 2017. Plants in Respiratory Disorders IIAntitussives, A Review. Journal of Pharmaceutical Research International, 16(3):1-21.

DOI: 10.9734/BJPR/2017/32974

Adomou AC, Yedomonhan H, Djossa B, Legba SI, Oumorou M, Akoegninou A. 2012. Etude Ethnobotanique des plantes médicinales vendues dans le marché d'Abomey-Calavi au Bénin. International Journal of Biological and Chemical Sciences, 6(2):745-772. DOI: 10.4314/ijbcs.v6i2.18

Ahmed RH, Barakat TE, Elnour RO, Elrassoul HH. 2018. Potential Anti-pyretic Activity of Guiera senegalensis Leaves. Journal of Advanced Research in Pharmaceutical Sciences \& Pharmacology Interventions, 2(1):18-21.

Ajibesin KK, Ekpo BA, Bala DN, Essien EE, Adesanya SA. 2008. Ethnobotanical survey of Akwa Ibom State of Nigeria. Journal of Ethnopharmacology, 115(3): 387-408.

DOI: 10.1016/j.jep.2007.10.021. 
Alowanou GG, Olounlade AP, Azando EVB, Dedehou VFGN, Daga FD, Hounzangbeadote SM. 2015. A review of Bridelia ferruginea, Combretum glutinosum and Mitragina inermis plants used in zootherapeutic remedies in West Africa: historical origins, current uses and implications for conservation. Journal of Applied Biosciences, 87(1): 8003-8014. DOI: $10.4314 /$ jab.v87i1.4

Al-Snafi AE. 2017. Pharmacology and therapeutic potential of Euphorbia hirta (Syn: Euphorbia pilulifera)-A review. IOSR Journal of Pharmacy, 7(3): 7-20.

Al-Snafi AE. 2019. Iraqi medicinal plants with antiviral effect-A review. IOSR Journal of Pharmacy, 9(7): 57-75.

Arbab AH, Parvez MK, Al-Dosari MS, AlRehaily AJ. 2017. In vitro evaluation of novel antiviral activities of 60 medicinal plants extracts against hepatitis B virus. Experimental And Therapeutic Medicine 14: 626-634. DOI: 10.3892/etm.2017.4530.

Babalola IT, Adelakun EA, Wang Y, Shode FO. 2012. Anti-TB activity of Sterculia setigera Del., leaves (Sterculiaceae). Journal of Pharmacognosy and Phytochemistry, 1(3): 17- 21.

Bahmani M, Saki K, Shahsavari S, RafieianKopaei M, Sepahvand, Adineh A. 2015. Identification of medicinal plants effective in infectious diseases in Urmia, northwest of Iran. Asian Pacific Journal of Tropical Biomedicine, 5(10): 858-864.

Béné K, Camara D, Fofie NGBY, Kanga Y, Yapi AB, Yapo YC, Ambé SA, Zirihi GN. 2016. Étude ethnobotanique des plantes médicinales utilisées dans le Département de Transua, District du Zanzan (Côte d'Ivoire). Journal of Animal \& Plant Sciences, 27(2): 42304250.

Chassagne F. 2017. Cancer du foie au Cambodge: état des lieux épidémiologiques, description des médecines traditionnelles utilisées et évaluation d'espèces médicinales sélectionnées. Thèse $\mathrm{PhD}$, Université de Paul Sabatier Toulouse III, France.

Chedi BAZ, Mandawari FK. 2018. In vitro evaluation of antiasthmatic activity of ethanol leaf extract of Guiera senegalensis J.F. GMEL (Combretaceae). African Journal of Pharmacy and Pharmacology, 12(9): 112-120. DOI: 10.5897/AJPP2017.4882.

Diatta W, Fall AD, Dieye AM, Faty S, Bassene E, Faye B. 2007. Experimental evidence of against cough activity of total alkaloids from Guiera senegalensis Lam. in guinea pig. Dakar medical, 52(2): 130-134.

Diop EA, Queiroz EF, Kicka S, Rudaz S, Diop T, Soldati T, Wolfender JL. 2018. Survey on medicinal plants traditionally used in Senegal for the treatment of tuberculosis (TB) and assessment of their antimycobacterial activity. Journal of Ethnopharmacology, 216: 71-78. DOI: https://doi.org/10.1016/j.jep.2017.12.037

Gyuris A, Szlavik L, Minarovits J, Vasas A, Molnar J, Hohmann J. 2009. Antiviral activities of extracts of Euphorbia hirta L. against HIV-1, HIV-2 and SIVmac251. International Journal of Experimental and Clinical Pathophysiology and Drug Research, 3(3): 429-432.

Guinnin FF, Sacramento TI, Sezan A, Ategbo JM. 2015. Etude Ethnobotanique des plantes médicinales utilisées dans le traitement traditionnel des hépatites virales $\mathrm{B}$ et $\mathrm{C}$ dans quelques départements $\mathrm{du}$ Bénin. International Journal of Biological and Chemical Sciences, 9(3): 1354-1366.

DOI: http://dx.doi.org/10.4314/ijbcs.v9i3.20.

Hossain MS, Uddin MS, Kabir MT, Begum MM, Koushal P, Herrera-Calderon O. 2017. In vitro screening for phytochemicals and antioxidant activities 
of Syngonium podophyllum L.: An incredible therapeutic plant. Biomedical and Pharmacology Journal, 10(3):126777.

DOI:

http://dx.doi.org/10.13005/bpj/1229.

Irie-N'guessan AG, Kablan BJ, KouakouSiransy NG, Leblais V, Champy P. 2011. Evaluation de la toxicité de cinq plantes antiasthmatiques de la médecine traditionnelle ivoirienne. International Journal of Biological and Chemical Sciences, 5(3): 1316-1319. DOI: 10.4314/ijbcs.v5i3.72285

Jdaidi N, Hasnaoui B. 2016. Étude floristique et ethnobotanique des plantes médicinales au nord-ouest de la Tunisie : cas de la communauté d'Ouled Sedra. Journal of Advanced Research in Science and Technology, 3(1): 281-291.

Jigam AA, Akanyan HO, Dauda BE, Ogbadoyi EO. 2011. Antiplasmodial, analgesic and antiinflammatory effects of crude Guiera senegalensis J. F. Gmel (Combretaceae) leaf extracts in mice infected with Plasmodium berghei. J. Pharmacogn. Phytother., 3(10): 150-154. DOI: 10.5897/JPP11.033

Jose RA, Kayode J. 2009. The Effect of Bridelia ferruginea Bark extracts on some pathogenic micro-organisms. Ethnobotanical leaflets, 8(1): 1042-1046.

Koné MW, Atindehou KK, Téré H, Traoré D. 2002. Quelques plantes médicinales utilisées en pédiatrie traditionnelle dans la région de Ferkessédougou (Côte d'Ivoire). Bioterre, 30: 30-36.

Kumar S, Malhotra R, Kumar D. 2010. Euphorbia hirta: Its chemistry, traditional and medicinal uses, and pharmacological activities. Pharmacognosy reviews, 4(7): 58-61. DOI: 10.4103/0973-7847.65327

Lamien CE, Meda A, Couacy-Hymann E, Ouedraogo AG, Nacoulma OG. 2005. The phytochemical composition and in vitro antiviral activity of decoctions from galls of Guiera senegalensis J.F. Gmel (Combretaceae) and their relative nontoxicity for chickens. Onderstepoort Journal of Veterinary Research, 72:111118.

Luka MI, Onuoha SC, Oladele VO, Aguiyi J. 2020. Phytochemical screening and in vitro evaluation of antibacterial activity of aqueous and ethanolic extracts of root and stem bark of Bridelia ferruginea. Benth. (Euphorbiaceae). Journal of Medicinal Plants Research, 14(1): 54-61. DOI: $10.5897 / J M P R 2019.6799$.

Mann A. 2012. Evaluation of antimicrobial activity of Anogeissus leiocarpus and Terminalia avicennioides against infectious diseases prevalent in hospital environments in Nigeria. Journal of Microbiology Research, 2(1): 6-10.

DOI:

10.5923/j.microbiology.20120201.02

Mann A, Ibrahim K, Oyewale AO, Amupitan JO, Fatope MO, Okogun JI. 2012. Isolation and elucidation of three triterpenoids and its antimycobacterial activity of Terminalia avicennioides. American Journal of Organic Chemistry, 2(2): 14-20.

DOI: 10.5923/j.ajoc.20120202.03.

Mann A, Ibrahim K, Oyewale AO, Amupitan JO, Okogun JI. 2009. Antimycobacterial activity of some medicinal plants in Niger State, Nigeria. African Journal of Infectious Diseases, 3(2): 44-48. DOI: 10.4314/ajid.v3i2.55080.

Mann A, Yahaya Y, Banso A, John F. 2008a. Phytochemical and antimicrobial activity of Terminalia avicennioides extracts against some bacteria pathogens associated with patients suffering from complicated respiratory tract diseases. Journal of Medicinal Plants Research, 2(5): 094-097.

Mann A, Amupitan JO, Oyewale AO, Okogun JI, Ibrahim K, Oladosu P, Lawson L, 
Olajide I, Nnamdi A. 2008b. Evaluation of in vitro antimycobacterial activity of Nigerian plants used for treatment of respiratory diseases. African Journal of Biotechnology, 7(11): 1630-1636. DOI: 10.5897/AJB08.438

Mann A, Amupitan JO, Oyewale AO, Okogun JI, Ibrahim K. 2007. An Ethnobotanical survey of indigenous flora for treating tuberculosis and other respiratory diseases in Niger State, Nigeria. Journal of Phytomedicine and Therapeutics, 12(1): 1-21. DOI: 10.4314/jopat.v12i1.41362

Nadembega P, Boussim JI, Nikiema JB, Poli F, Antognoni F. 2011. Medicinal plants in baskoure, kourittenga province, Burkina Faso: an ethnobotanical study. Journal of Ethnopharmacology, 133(2): 378-395. DOI: 10.1016/j.jep.2010.10.010.

Ndhlala AR, Amoo SO, Ncube B, Moyo M, Nair JJ, Van Staden J. 2013. Antibacterial, antifungal, and antiviral activities of African medicinal plants. In Medicinal plant research in Africa. Elsevier: Oxford ; 621-659. DOI: http://dx.doi.org/10.1016/B978-0-12405927-6.00016-3

Nwafor PA, Hamza HG. 2007. Antidiarrhoeal and anti-inflammatory effects of methanolic extract of Guiera senegalensis leaves in rodents. J. Nat. Remedies, 7(1): 72-79.

Organisation Ouest Africaine de la Santé (OOAS). 2013. Pharmacopée d'Afrique de l'Ouest. OOAS, $1: 268 \mathrm{p}$.

Owoseni AA, Ayanbamiji TA, Ajayi YO, Ewegbenro IB. 2010. Antimicrobial and phytochemical analysis of leaves and bark extracts from Bridelia ferruginea. African Journal of Biotechnology, 9(7): 1031-1036.

Parvez MK, Al-Dosari MS, Arbab AH, AlRehaily AJ, Abdelwahid MA. 2020. Bioassay-guided isolation of anti- hepatitis B virus flavonoid myricetin-3O-rhamnoside along with quercetin from Guiera senegalensis leaves. Saudi Pharmaceutical Journal, 28(5): 550-559. DOI: https://doi.org/10.1016/j.jsps.2020.03.00 6.

Prachi S, Pradeep T. 2014. 13 $\alpha$-methyl-27norolean-14-en-3 $\beta$-ol, a Triterpene isolated from the stem of Euphorbia Hirta (Linn) Possess an Anti-Asthmatic Properties. Research Journal of Chemical Sciences, 2231: 606X.

Sanogo, R. 2012. Guiera senegalensis J.F. Gmel. In Medicinal Plants/Plantes Médicinales, Schmelzer GH, GuribFakim A (Ed.). Wageningen, Pays Bas.

Tine Y, Diop M, Ndoye I, Diallo A, Wele A. 2019. Revue bibliographique sur la composition chimique et les activités biologiques de Guiera senegalensis JF Gmel. (Combretaceae). International Journal of Biological and Chemical Sciences, 13(7): 3449-3462. DOI: https://dx.doi.org/10.4314/ijbcs.v13i7.37

Tor-Anyiin TA, Akpuaka MU, Oluma HOA. 2011. Phytochemical and antimicrobial studies on stem bark extract of Sterculia setigera, Del. African Journal of Biotechnology, 10(53): 11011-11015. DOI: $10.5897 / A J B 10.1493$.

Usman H, Kaigama AU, Ibisagba OO, Fulata AM, Ahmed IA. 2018. Phytoconstituents evaluation and antimicrobial efficacy of the crude flavonoids and saponins root bark extracts of Terminalia avicennioides and Ficus polita. Journal of Herbmed Pharmacology, 7(2): 106-111. DOI: 10.15171/jhp.2018.18.

Van der Kooyn F, Sullivan SE. 2013. The complexity of medicinal plants: The Traditional Artemisia annиa formulation, current status and future perspectives, Journal of Ethnopharmacology, 150(1): 1-13. DOI: 10.1016/j.jep.2013.08.021. 
World Health Organization (WHO). 2004.

SARS: clinical trials on treatment using a combination of traditional Chinese medicine and Western medicine: report of the WHO International Expert Meeting to review and analyse clinical reports on combination treatment for SARS, 8-10 October 2003. WHO, Beijing, People's Republic of China.

Willcox M, Sanogo R, Diakite C, Giani S, Paulsen BS, Diallo D. 2012. Improved traditional medicines in Mali. The Journal of Alternative and Complementary Medicine, $\mathbf{1 8}(3)$ : 212-220. DOI: https://doi.org/10.1089/acm.2011.0640.

Yapi AB, Zirihi GN. 2015. Etude ethnobotanique des Asteraceae médicinales vendues sur les marchés du district autonome d'Abidjan (Côte
d'Ivoire). International Journal of Biological and Chemical Sciences, 9(6): 2633-2647. DOI: 10.4314/ijbcs.v9i6.10

Yeo D, N'guessan JD, Sea T, Coulibaly YA, Djaman AJ, Tako NA, Yavo JC, GuedeGuina F. 2008. Évaluation de l'activité antiasthmatique et antitussive de Combretum molle: plante médicinale de la pharmacopée ivoirienne. Phytothérapie, 6(6): 348-351. DOI : 10.1007/s10298-008-0347-6

Zerbo P, Millogo-Rasolodimey J, NacoulmaOuedraogo OG, Van Damme P. 2007. Contribution à la connaissance des plantes médicinales utilisées dans les soins infantiles en pays San, au Burkina Faso. International Journal of Biological and Chemical Sciences, 1(3): 262-274. DOI : 10.4314/ijbcs.v1i3.39704. 